\title{
Innovative use of artificial intelligence in urogynecology
}

\author{
Jouhayna Bentaleb ${ }^{1}$ - Maryse Larouche ${ }^{2,3}$
}

Received: 14 January 2020 / Accepted: 15 January 2020 / Published online: 13 February 2020

(C) The International Urogynecological Association 2020

Development of artificial intelligence (AI) has become a worldwide priority in many fields. AI consists in the use of complex algorithms in order for machines to reason and perform cognitive functions, including problem-solving, outcome prediction, and decision-making. In obstetrics and gynecology, some innovative advances have been published with varying success, including automated fetal intrapartum surveillance reading, as well as in vitro fertilization success prediction tools and embryo selection models [1]. Urogynecology, in particular, will benefit greatly from future developments in AI.

First, developments in AI are enabling advances in telemedicine. Through the use of wearable devices linked to AI systems, patients' conditions could be monitored, tracked, and managed virtually. Currently, electronic medical records software automates scheduling between caregivers, organizes care plans, creates alerts for follow-ups, and automates billing, as well as portals for patients and families. Telemedicine use is also increasing through paid applications that can be linked with electronic medical records, and could be further tailored to the urogynecological aging population. Virtual visits allow for follow-ups that do not require a physical examination, and can shorten wait times while avoiding transportation difficulties for those patients living afar or with limited mobility. Telemedicine in urogynecology could exploit the use of wearable devices, such as those monitoring for urinary incontinence (which could be more precise than bladder diaries), and post-void residual bladder volume scanners. Wearable devices monitoring bladder volumes have recently been

Maryse Larouche

maryse.larouche@gmail.com

1 Department of Obstetrics and Gynecology, University of Montreal, Montreal, QC, Canada

2 St. Mary's Research Centre, Montreal, QC, Canada

3 Department of Obstetrics and Gynecology, McGill University, Montreal, QC, Canada developed for use in pediatric populations with nocturnal enuresis and other types of urinary incontinence [2]. Treating teams could remotely access collected data to manage patients, or ultimately, this could be done by an AI system.

Another extremely valuable application of AI in urogynecology would involve complex management algorithms, to help patients and providers to navigate through available options and predict response to treatment for women with various pelvic floor disorders. The counseling role of urogynecologists, gynecologists, and female urologists is complicated by the fact that multiple pathological conditions of the pelvic floor frequently co-exist, and a series of patient factors can impact outcomes. Despite these limitations, randomized controlled trials (RCT) results are frequently cited to patients during counseling as the only available estimate of outcomes. Recently, female pelvic reconstructive surgery management algorithms have been developed. For example, Jelovsek et al.'s algorithm predicts the risk of de novo stress urinary incontinence after prolapse surgery [3]. However, the algorithm, based on RCT data, was found to have limited external generalizability, as demonstrated by the lack of improvement in patient satisfaction, with the decision to place a mid-urethral sling when women were randomized to using the algorithm versus not [4], and limited predictive value when used as predictive diagnostic test [5]. A more recent algorithm, also developed by Jelovsek et al., and based on RCT data, estimates risk of recurrence, complications, and health outcomes after prolapse repair [6]. Optimally, in the future, an all-encompassing algorithm would be developed to propose complete patient-centered solutions tailored to each woman's characteristics, symptomatology, and expected goals for outcomes. AI could exploit big data, including patientreported outcomes, to allow the development of such an algorithm.

Intrinsic and extrinsic challenges affect the progress and usability of AI in urogynecology, as well as in medicine overall, including new knowledge needed to be acquired, new types of errors that can occur (including the algorithm not predicting the outcome appropriately), confidentiality and ethical issues, the matter of intellectual property, as well as the 
expensive funding required to develop those technologies. Development of guidelines for regulatory approval of algorithms prior to widespread adoption could protect both patients and physicians [7].

Artificial intelligence will likely occupy a pivotal role in the future of female pelvic medicine and reconstructive surgery, and will no doubt evolve rapidly. Care providers will have to learn to introduce these new tools into practice, while maintaining a human, caring approach to patient care.

\section{References}

1. Emin EI, Emin E, Papalois A, Willmott F, Clarke S, Sideris M. Artificial intelligence in obstetrics and gynaecology: is this the way forward? In Vivo. 2019;33(5):1547-51.

2. Van Leuteren PG, Nieuwhof-Leppink AJ, Dik P. SENS-U: clinical evaluation of a full-bladder notification - a pilot study. J Pediatr Urol. 2019;15(4):381.e1-5.
3. Jelovsek JE, Chagin K, Brubaker L, et al. A model for predicting the risk of de novo stress urinary incontinence in women undergoing pelvic organ prolapse surgery. Obstet Gynecol. 2014;123(2 Pt 1): 279-87.

4. Miranne JM, Gutman RE, Sokol AI, Park AJ, Iglesia CB. Effect of a new risk calculator on patient satisfaction with the decision for concomitant Midurethral sling during prolapse surgery: a randomized controlled trial. Female Pelvic Med Reconstr Surg. 2017;23(1):17-22.

5. Sabadell J, Salicrú S, Montero-Armengol A, Rodriguez-Mias N, GilMoreno A, Poza JL. External validation of de novo stress urinary incontinence prediction model after vaginal prolapse surgery. Int Urogynecol J. 2019;30(10):1719-23.

6. Jelovsek JE, Chagin K, Lukacz ES, et al. Models for predicting recurrence, complications, and health status in women after pelvic organ prolapse surgery. Obstet Gynecol. 2018;132(2):298-309.

7. Greenfield D. Artificial intelligence in medicine: applications, implications, and limitations. http://sitn.hms.harvard.edu/flash/2019/ artificial-intelligence-in-medicine-applications-implications-andlimitations/. Published 2019. Accessed 8 Jan 2020.

Publisher's note Springer Nature remains neutral with regard to jurisdictional claims in published maps and institutional affiliations. 